\title{
Degradation of Unplasticised Poly Vinyl Chloride Based Engineering Products Upon Exposure to Ultra Violet Radiation
}

\author{
K. G. Alahapperuma and A. M. P. B. Samarasekara ${ }^{1 *}$ \\ Department of Materials Science and Engineering \\ University of Moratuwa \\ Sri Lanka
}

\begin{abstract}
Poly vinyl chloride (PVC) is considered as a widely used polymer for various applications. It is durable, inexpensive and resistant to heat, water and chemicals. Additives such as lubricants, heat stabilizers, plasticizers, impact modifiers, fillers and ultra violet (UV) stabilizers enhance its durability and make it suitable for a range of applications. PVC is used for automobile and other sectors in products ranging from piping and siding, blood bags and tubing, to wire and cable insulation applications. Poly vinyl chloride exhibits high sensitivity to UV rays and it is a life time deciding factor specially in outdoor applications. This study was conducted to analyse the degradation behavior of PVC under the UV environment. Commercially available $32 \mathrm{~mm}$ PNT7 unplasticised poly vinyl chloride (uPVC) pipes were selected for this research. Initially, tensile strength, elongation, hardness and water absorption properties were analysed. Ultra violet ray exposure test was performed for PVC samples to evaluate the photo-degradation behavior at selected UV exposure time periods. Tensile strength, elongation, hardness, water absorption properties and visual inspection tests were performed after the UV exposure test. Scanning electron microscopic images and differential thermal analysis were also used to analyze the degradation behavior. It was indicated a slight reduction of tensile strength, percentage elongation and hardness of samples with increase of $U V$ exposure time. Scanning electron microscopic images of products showed degraded features after UV exposure test. This study revealed that uPVC based products are resistant to photodegradation by $U V$ rays.
\end{abstract}

Keywords: Photodegradation, $U V, u P V C$, degradation

\section{INTRODUCTION}

Polymers are high molecular weight materials or macromolecules made up of many repeating chemical units. The term polymer can be defined as a macromolecule with high molecular mass arising due to the joining of large number of smaller molecules called monomers. A different monomer or combination of monomers is used to manufacture each different type or family of polymers. There are many polymers around us that are so familiar to us. The polymers are characterized by variable molecular weight (depending on the source or mode of synthesis or extraction), low specific gravity, better resistance to erosion and corrosion, insects or fungi etc. The advantageous aspect of polymeric materials over other materials as per our property requirements may be soft to rigid or tough, transparent to opaque, light to heavy, crystalline to amorphous (Samarasekara and Jayasuriya, 2014).

Department of Materials Science and Engineering, University of Moratuwa, Sri Lanka

* Corresponding author: banduamp@gmail.com

This is an open-access article distributed under the terms of the Creative Commons Attribution License, which permits unrestricted use, distribution, and reproduction in any medium, provided the original author and source are credited. 
Different monomers or combination of monomers are used to manufacture different type or family of polymers. Polymers are classified based on their origin as natural and synthetic polymers. Natural polymers are the ones which are isolated from the natural sources. Cotton, silk, wool, natural rubber etc. belong to this type. There are also polymers modified from these natural polymers viz., cellophane, cellulose, chitosan, rayon, leather etc. Synthetic polymers are the polymers based on the low molecular weight compounds such as polyethylene, polyvinyl chloride (PVC), nylon, bakelite etc. Polymers have a great influence on our society today (Umadaran et al., 2016).

PVC is one of the mostly used polymeric materials among the plastic materials used today (Geyer et al., 2017). It is mixed with plasticizers that soften the plastic so that it is more pliable. If plasticizers were not added, the plastic would be hard and brittle. Some of the plasticizers used in vinyl plastics are phthalate esters. These esters are volatile compounds of low molecular weight (Wahl et al., 1999). PVC is used extensively across a broad range of industrial, technical and everyday applications including widespread use in building, transport, packaging, electrical/electronic and healthcare applications. PVC is a very durable and longlasting material which can be used in a variety of applications, either rigid (uPVC) form or flexible (PPVC) (Titow, 2012). The original properties of PVC change due to different environmental factors and these changes lead to a decrease in the polymer's molecular weight and physical and chemical properties. Ultraviolet radiation is one source that causes changes in the molecular structure of PVC. Under the influence of incident energetic radiation, photochemical processes of PVC occur such as chain scission. These changes decrease the performance of PVC based products and ultimately fracture occurred (Hawkins,1984).Therefore, it is necessary to analyse the degradation behavior of PVC under the UV environment. The objective of this research is to analyse the photodegradation of PVC based products by varying the UV exposure time to get an idea of the degradation process during the service life.

\section{METHODOLOGY}

Commercially available $32 \mathrm{~mm}$ uPVC $\mathrm{PNT}_{7}$ pipes (five samples) were selected for this analysis.

\section{Characterization of UPVC pipes}

The UPVC pipes were characterized using images taken Scanning Electron Microscope (SEM), FTIR spectroscopic techniques and Thermogravimectric Analysis (TGA).

\section{Morphological investigation of UPVC pipes}

The surface morphology of UPVC pipes were investigated by SEM (CARLZIESS EBO 18) before and after the UV exposure test.

\section{Thermogravimetric analysis (TGA)}

Thermal stability of the PVC samples was determined by TGA analysis. The sample was placed in an instrument and heated with rate of $10^{\circ} \mathrm{C} / \mathrm{min}$ at the temperature range between $25{ }^{\circ} \mathrm{C}-600{ }^{\circ} \mathrm{C}$ under high purity nitrogen environment using TA Instruments-SDT Q600 machine. 


\section{FTIR spectroscopy}

The original and degraded samples were investigated by FTIR spectroscopy using a BRUKER Alpha-E spectrophotometer. The samples were investigated under ATR mode of the spectrometer. The FTIR spectra of the samples were obtained in the transmittance mode in the range of $4000-500 \mathrm{~cm}^{-1}$.

\section{Testing of samples}

Initially, original tensile strength, percentage elongation, hardness (Shore D) and water absorption properties were measured. After that prepared samples were subjected to the UV exposure test under the standard conditions. Seven set of samples were removed from the UV chamber at different time intervals of 24, 48, 72, 96, 120, 144 and 168 hours. Tensile strength, percentage elongation, hardness, water absorption properties and visual inspection tests were done after the UV exposure treatment. Additionally, FTIR spectroscopy, TGA analysis and scanning electron microscopic (SEM) images were also obtained. Obtained properties were compared with the properties of original (reference) set of samples. Accelerated UV exposure test was performed as per ISO: 4582 standards. Tensile properties, hardness and water absorption properties were performed under ASTM D 638, ASTM D 2240 and ISO 62 standards respectively. All tests were performed for the reference sets of samples under mentioned standard conditions. Results of each test obtained after different exposure time to UV were compared with the corresponding test results of reference samples.

\section{RESULTS AND DISCUSSION}

\section{Characterization of UPVC samples}

\section{Morphological investigation}
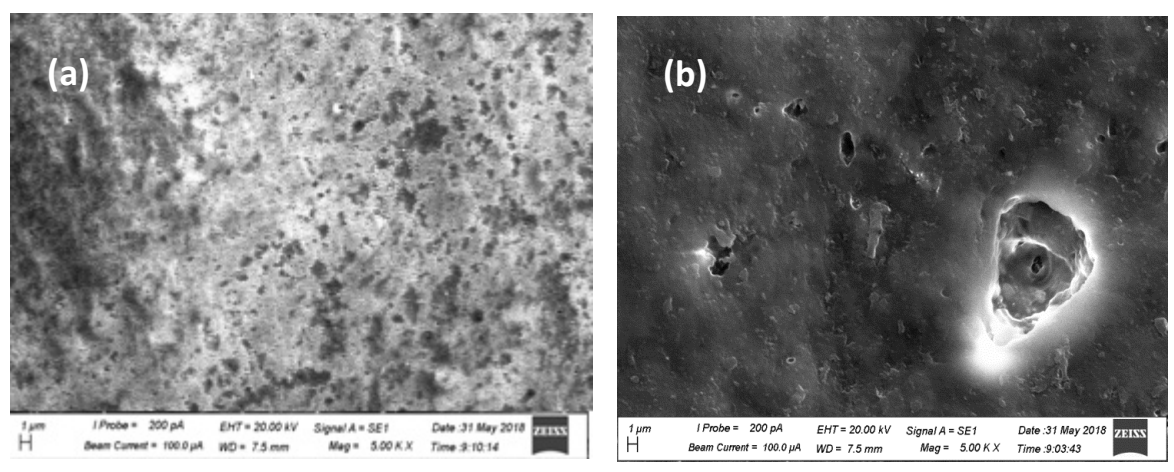

Figure 1. SEM images of (a) reference sample (b) after exposing to UV for 168 hours.

In comparison to reference sample, few degraded signs were found in the SEM image of the specimen exposed to UV for 168 hours (Figure 1) indicating the surface deterioration due to photo-degradation during the UV exposure test. 


\section{Analysis of FTIR spectra}

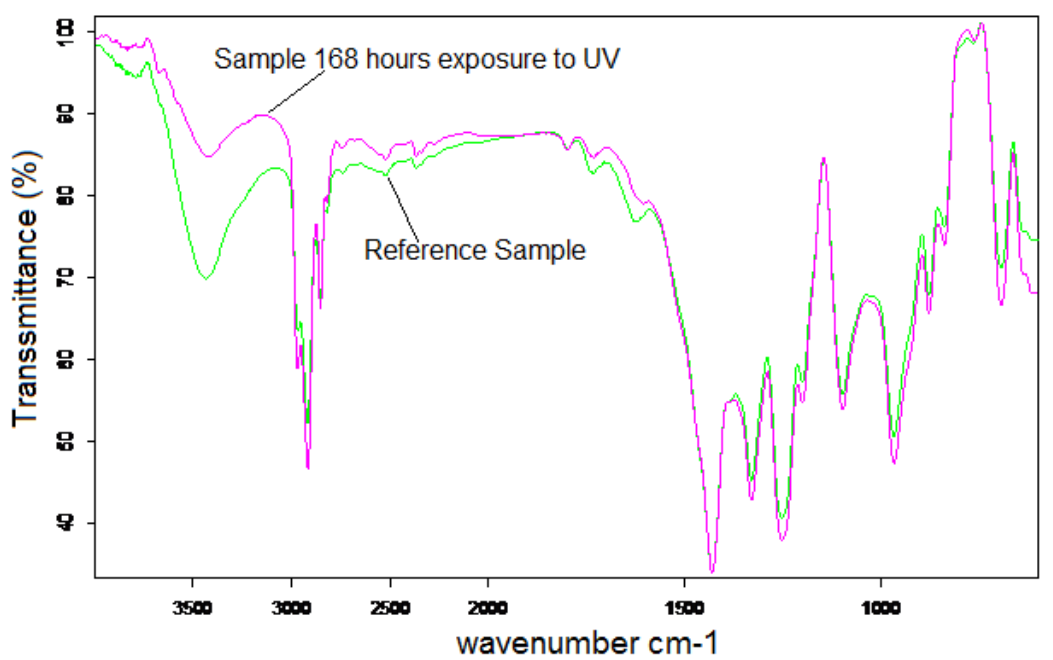

Figure 2. FTIR spectrums of reference sample and samples exposed to UV up to 168 hours.

According to the Figure 2, the FTIR spectrums of reference sample and samples exposed to UV for 168 hours did not show the significant structural changes due to UV exposure.

\section{Thermogravimetric analysis}

Thermal analysis test results showed that degradation of the reference sample started at $253{ }^{\circ} \mathrm{C}$ and degraded sample after 168 hours exposed to UV showed the same property at $250.1^{\circ} \mathrm{C}$. Degraded sample indicated slightly lower thermal stability than the reference sample, indicating internal structural break down due to photo-degradation.

\section{Analysis of Mechanical Properties}

\section{Tensile Strength}

Tensile test results in Figure 3, showed the gradual reduction of tensile strength with increase of UV exposure time. Gradual reduction of tensile strength of samples may be due to the reduction of molecular-chain stiffness, caused by chain scissions due to UV attack. 


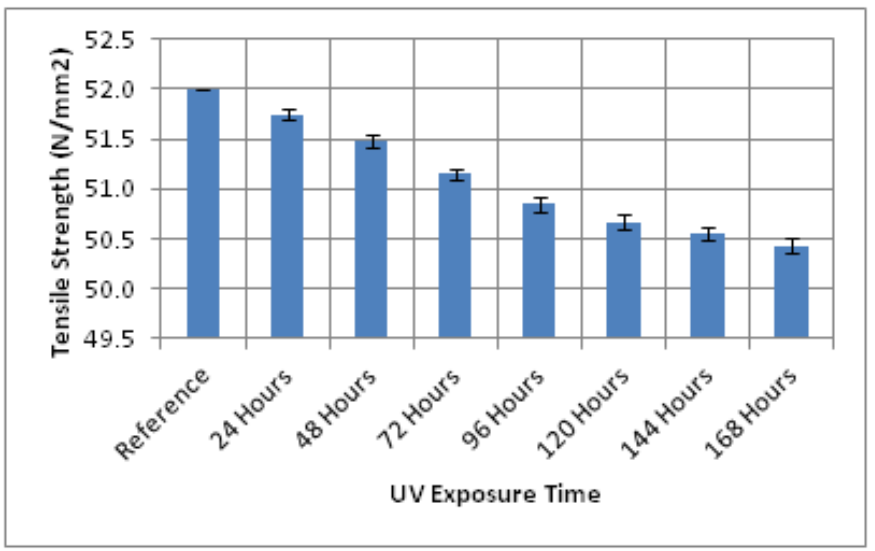

Figure 3. Tensile strength of reference sample and variation with exposure time UV.

\section{Elongation at Break}

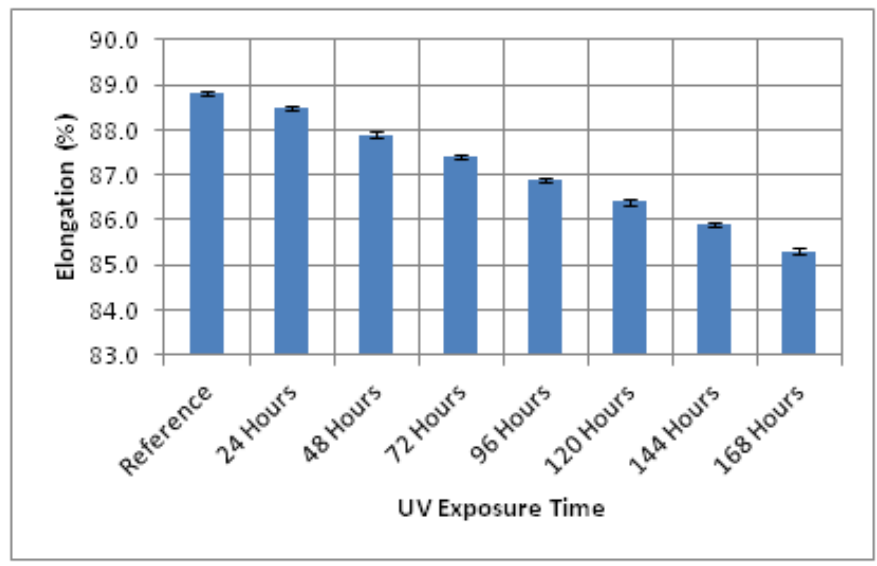

Figure 4. Relative elongation of sample with exposure time of UV.

Test results of percentage elongation at break also reduced somewhat gradually and slightly with the increase of UV exposure time (Figure 4). The causes of reduction of tensile strength could have also attributed for a gradual reduction in the elongation of sample. During the degradation, structure becomes embrittled and molecular weight reduced.

\section{Hardness Properties}

According to the Figure 5, there is a gradual reduction of hardness with increase of UV exposure time. Reduction of hardness may be due to the reduction of structural stiffness or increase of surface softness caused by the degradation process. 


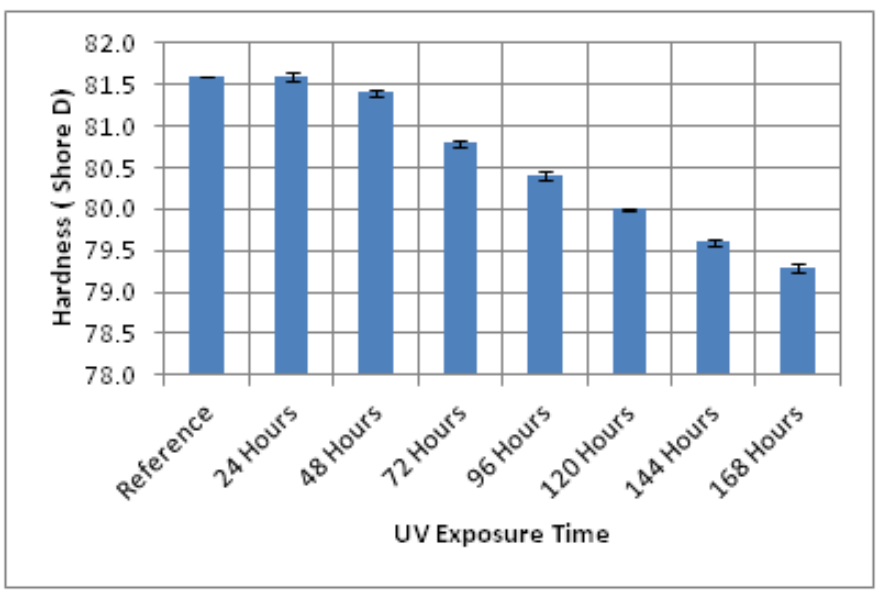

Figure 5. Hardness of sample with exposure time of UV.

\section{Water Absorption Properties}

Figure 6 indicated that there is a gradual increase of water absorption with increase of UV exposure time. This increase could be due to the formation of surface cavities or cracks, due to the formation of loose surface layer during the degradation process.

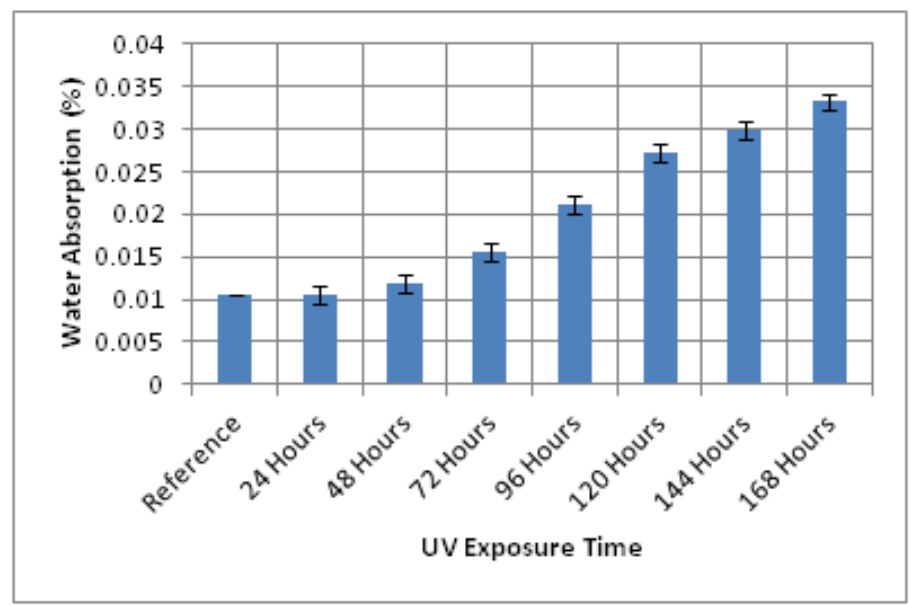

Figure 6. Water absorption of sample with exposure time of UV.

\section{CONCLUSIONS}

According to the experimental results, it can be concluded that tested samples did not show significant mechanical property variation after the photodegradation. However, there were surface morphological changes and reduction of thermal stability of the product due to photodegradation process. 


\section{REFERENCES}

Geyer, R., Jambeck, J.R. and Law, K.L. (2017). Production, use, and fate of all plastics ever made. Science advances. 3(7), e1700782.

Hawkins, W.L. (1984). Polymer degradation. pp 3-34. In: Harwood, H. J. (Ed.) Polymer Degradation and Stabilization. Springer, Berlin, Heidelberg.

Samarasekara, A.M.P.B. and Jayasuriya, E.A.P.C.D. (2014). February. Synthesis of Biodegradable Polyolefins Based Polymer Composites Using Degradable Natural Materials. In Proceedings of International Forestry and Environment Symposium. 18.

Titow, M.V. (2012). PVC technology. Springer Science \& Business Media.

Umadaran, S., Somasuntharam, P. and Samarasekara, A.M.P.B. (2016). April. Preparation and characterization of Cellulose and Hemi-Cellulose based degradable composite material using sugarcane waste. In Moratuwa Engineering Research Conference (MERCon), 2016. 367-372. IEEE.

Wahl, H.G., Hoffmann, A., Häring, H.U. and Liebich, H.M. (1999). Identification of plasticizers in medical products by a combined direct thermodesorption-cooled injection system and gas chromatography-mass spectrometry. Journal of Chromatography. 847(1-2), 17. 